Document downloaded from:

http://hdl.handle.net/10251/143321

This paper must be cited as:

Grillo-Espinoza, H.; Alemany Díaz, MDM.; Ortiz Bas, A. (09-2). Modelling Pricing Policy Based on Shelf-Life of Non Homogeneous Available-To-Promise in Fruit Supply Chains. IFIP Advances in Information and Communication Technology. 480:608-617. https://doi.org/10.1007/978-3-319-45390-3_52

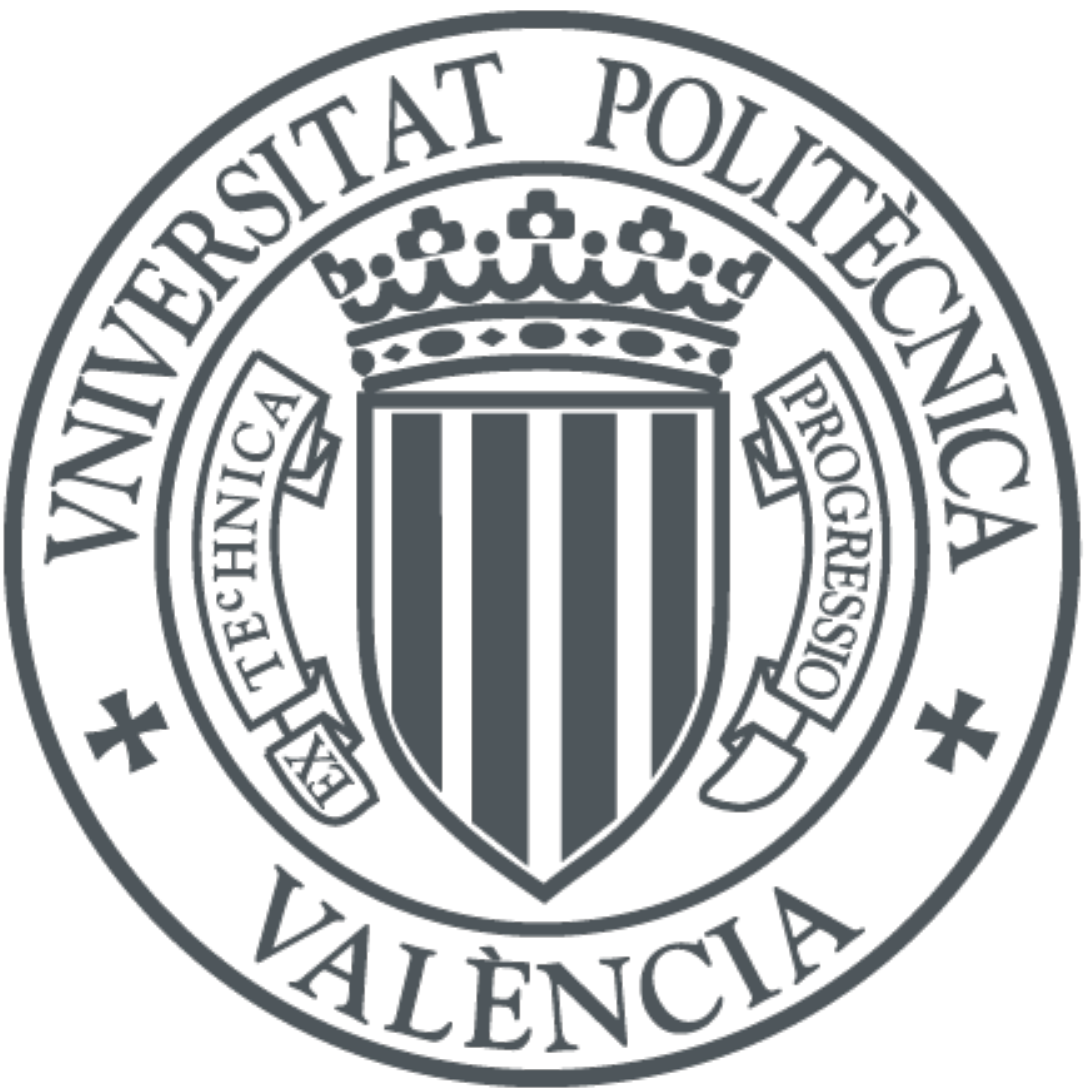

The final publication is available at

https://doi.org/10.1007/978-3-319-45390-3_52

Copyright Springer

Additional Information 


\title{
Modelling Pricing Policy based on Shelf-life of Non Homogeneous Available-To-Promise in Fruit Supply Chains
}

\author{
Hanzel Grillo*, M.M.E Alemany, A. Ortiz \\ Centro de Investigación de Gestión e Ingeniería de Producción (CIGIP), Universitat Politècnica \\ de València, Camino de Vera S/N, 46022, Valencia, Spain.
}

\begin{abstract}
Fruit Supply Chains (SCs) are influenced by uncontrollable natural factors causing heterogeneity in their products, as regards certain attributes that are relevant to customers and vary over time because of the shelf-life. As a consequence customers should be served not only with the required quantity and due date as usual, but also with the quality, freshness and homogeneity specified in their orders. The order promising process (OPP) is based on the uncommitted availability of homogeneous product quantities in planned lots (ATP) that are uncertain. Therefore, there is a risk of not being reliable in the commitments because of discrepancies between the real and planned homogeneous quantities. Furthermore, due to the shelflife (SL), serving customers with the freshest product introduce the risk of increasing waste because of the aging process. To efficiently manage these risks, this work proposes a mathematical model for handling the heterogeneous ATP in fruit SCs and a pricing policy based on the product SL in the moment of delivery. In order to illustrate the application of the modelling approach, a short numerical example is introduced. The example evidences a conflictive situation when optimizing the assignation of homogeneous ATP between serving orders with fresh and more valuable product, what could lead to increase the risk of having waste because of expiration, and consequently, more costs and less profit.

Keywords: Order-promising process, Available-To-Promise, Lack of Homogeneity in the Product, fruit supply chain, perishability.
\end{abstract}

\section{Introduction}

The order promising process (OPP) presents especial features and more complexity in fruit supply chains (SCs), where uncontrollable natural factors like weather, land, water drought, etc. are present. That kind of factors can confer heterogeneity to units of the same product as regards certain attributes relevant to customers. Additionally, due to the perishability, products are not just affected by the non homogeneity but also for the aging process. The order proposals of these SCs require homogeneity in

Corresponding author

E-mail address: hangries@ upvnet.upv.es / grillo7@gmail.com (Hanzel Grillo) 
units of the same product, and normally, customers expect to be served with the freshest product, with the quality required, but with a reasonable price. For this reason, the pricing policy can be directly based on the status of the product at the time of delivery. These aspects become a problem when customer orders are promised based on future planned homogeneous quantities; because final characteristics are not known certainly until the product is harvested, classified, packed and transported to the customer. Then, fruit supply chains has inherent uncertainty in the handling of the available to promise (ATP) quantities, due to not just the homogeneity in the product, but also to the shelf life (SL). One of the main challenges when modelling the OPP in fruit SCs, is firstly to estimate the homogeneous ATP quantities derived from the master plan in advanced to be produced and classified. Secondly, due to perishability, those homogeneous ATP quantities deteriorate over time being necessary to provide traceability. Thirdly, the harvesting time and SL, become critically important in terms of the freshness of the product in the moment of delivery. Consequently, it also affects earns obtained, in case the pricing policy is directly linked with the product freshness. Therefore, the main contribution of this paper is to include these challenges combined into the OPP for fruit SCs. To properly handle them, a mathematical programming model for supporting the OPP has been formulated that takes into account the homogeneity required in the ATP, the dynamism conferred to it by the SL and a proper pricing policy linked to the perishability of the product.

The rest of the paper is organized as follows: Section 2 presents a short summary of the related work founded. Section 3 and 4 describes the proposed way to model the ATP in fruit SCs and the modelling of the SL, respectively. Section 5 presents the link assumed between the remaining SL and the pricing policy for the ATP. Section 6 presents a numerical example to show the conflict when freshness and benefit are both them combined for being optimized. Finally, Section 6 presents the main conclusions and future research lines.

\section{Related Work}

This work aims to introduce less investigated characteristics in the OPP, such as the homogeneous ATP quantities and their perishability in fruit SCs. The homogeneous ATP concept is included in the so called lack of homogeneity in the product (LHP). LHP is defined as the absence of uniformity among units of the same product, that can originate from the incorporation of raw materials directly derived from nature, and/or production processes features that confer heterogeneity to it (Alarcon et al. 2011, Alemany et al. (2013, 2015), Mundi et al.(2015) and Grillo et al. 2016). Improper management of non-homogeneous units of the same product and its inherent uncertainty can impact very negative on customer service level. It is established that, due to inherent LHP uncertainty, the planned size of homogeneous sublots and the real ones can differ once produced and classified into subtypes. A variety of other studies, like Lin et al. (2010), have dealt with some characteristics related with the LHP without explicitly mention it. In the specific case of fruit SCs, the lack of literature is even more evident, being Kilic et al. (2010) the closest case validated in the food processing sector. In this paper, the main LHP characteristic appear 
randomly in raw materials with a strong effect over the finished product. The perishability (SL consuming) is identified as one of LHP types (Grillo et al., 2016), and as a cause of conflictive decisions in order to either maximise the incomes by sells of the product, or minimise the cost as a consequence of avoiding waste. Since customers require homogeneous and fresh products, they hope the price is directly linked on its quality, homogeneity and its freshness (that can be measured as a fraction of consumption of its maximum SL). This is also a lacking area of research because of only a few models have dealt with pricing of perishables (see for example Maihami and Karimi(2014) and Tsao and Sheen(2008)). Furthermore no one makes it under LHP conditions. The findings from the literature, that serve as a basis for the main contribution of this work could be summarised as follows: (i) when dealing with perishables, there is a lack of literature dealing with OPP in LHP contexts that consider the splitting of ATP into homogeneous quantities; (ii) since customers of fruit SCs specify the subtypes required in their orders, it is necessary to estimate not only the homogeneous ATP quantities, but also the subtype obtained to accomplish with customer requirements; (iii) existing OPP models for fruit SCs do not calculate the remaining SL of homogeneous ATP quantities, in a way that the selling price could be linked to it. The above issues confer specific characteristics to the OPP that in case of not being correctly managed could originate: a) dissatisfaction of customers due to the failure in the freshness desired, and b) even more, high stocks and waste that produces SC inefficacy and loss of profit.

In the next sections the proposed way to characterise homogeneous ATP in fruit SCs is presented, followed by the proposed calculation of the SL. Finally, the combination of both is made to properly model the pricing policy for the homogeneous ATP, based on the SL of the product.

\section{Modelling the Homogeneous Available-To-Promise for Fruit Supply Chains}

With the aim of serving customers in the homogeneity terms required in their orders, fruit supply chains are forced to include different classification steps along the productive process (Blanco et al., 2005). Therefore, fruit is sorted into homogeneous sublots based on the following characteristics and/or attributes: (i)Variety: it represents the types of the same fruit; (ii) Quality: the product can be classified into quality categories, according to the status it brings from the fields; (iii) Calibre: this criteria refers to the size and volume of the product; (iv) Packaging type: it refers to the different boxing classes that the product can be commercialized and (v) Harvesting time: it represents the time period at which the fruit is collected from fields. Since each of this characteristic can have different values, the specific combination of each attribute can define what is known as a "subtype". Since these attributes can vary with the specific type of fruit, it will be always possible to define the different subtypes as a combination of them. In order to model the homogeneous Available-To-Promise in this kind of SCs, let us define the following nomenclature: 


\section{Indices}

p Production plant

$t$ Time buckets.

$h$ Harvesting time of the product available to promise.

$i$ The product.

$b$ Subtypes, defined as the combination of quality, calibre and packaging type.

$o$ Customer order proposals waiting to be promised.

$r$ Price range defined based on the shelf life.

\section{Parameters}

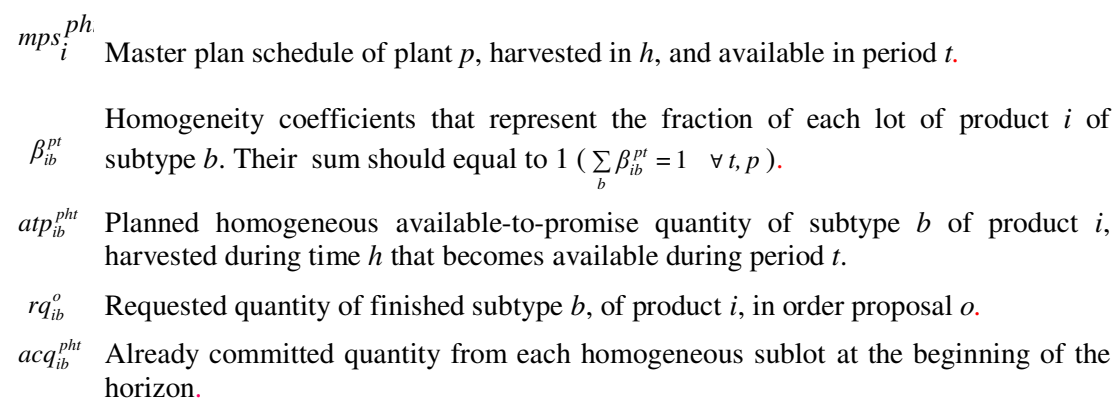

\section{Variables}

Binary variable with a value of 1 if requested quantity $r q_{i b}^{o z}$ is completely served by the $a t p_{i b}^{p h t}$ within price range $\mathrm{r}$, and a value of 0 otherwise.

$U A T P_{i b}^{p h t}$ The updated homogeneous $a t p_{i b}^{p h t}$ after taking into account the customer order proposals committed.

If a multi-plant production stage should serve different customers with the subtype specified in their orders on a required due date, and where the supply stage is taking into account the inclusion of the product's harvesting time, the first step to be done is ensuring a reliable calculation of homogeneous ATP quantities of each subtype. Since ATP quantities are derived from the subdivision of the master plan, through the fraction corresponding to each homogeneous subtype, and subtracting the requested quantity in the proposals that might be committed beforehand and the quantity promised to the incoming order proposals; this can be represented through Eq. (1):

$$
a t p_{i b}^{p h t}=m p s_{i}^{p h t} \beta_{i b}^{p t}-a c q_{i b}^{p h t}-\sum_{o} \sum_{r} r q_{i b}^{o} Y_{i b}^{o p h t r} \quad \forall p, h, t, i, b
$$

In order to ensure that that the ATP defined in Eq. (1) can be served just from the specific subtype required by the customer, and that different subtypes cannot be mixed the Eq.(2) must be considered:

$$
\sum_{b} Y_{i b}^{o p h t r}=1 \quad, \quad \forall o, p, h, t, r, i
$$


As it can be seen in Eqs. (1)-(2), the variable $Y_{i b}^{\text {ophtr }}$ depends on the price range inside the order proposal might be served. As a novel approach, we are connecting the price range of the served orders with the product SL at delivery time. In sections 4 and 5 we will introduce the concepts and explain how to model this approach.

\section{Modelling the Shelf-life}

One strategic issue on modelling the ATP for fruit supply chains is the perishability. This factor makes ATP characteristics of each subtype defined in Eq. (1) to change over time while the SL is consuming. If we assume that the SL is pre-defined for each subtype, the freshness of products committed with customers will depend on the remaining SL in the moment of delivery to customer. To maintain a traceability of the remaining SL is necessary in fruit SC for different reasons: 1) customer satisfaction is strongly influenced by the freshness of the product, 2) in case the freshness will be lower than expected, a discount in the price can restore a proper level of customer satisfaction, and finally, 3) because of the perishability, product waste should occur if its SL is exhausted and it has not been assigned to any customer order. Therefore, to mathematically describe the relationship of homogeneous ATP with its selling price, it is necessary to first calculate the SL. When reserving homogeneous ATP in a specific time period, to be delivered in the customer's place on a due date $\left(d d_{o}\right)$, the $\mathrm{SC}$ must take into account the time required for transporting the product from the harvesting point to the packing plants where the classifications and packing operations take place, as well as the transporting time since the product is shipped from the plant until it is delivered to the customer. This is necessary to deliver the product with the minimum portion of its $\mathrm{SL}\left(s l_{i b}\right)$ already consumed. In terms of calculation, we can define the fraction of already lost SL ( $L S L^{\circ}$ ), of the ATP in the moment of the delivery to the customer as follows:

$$
L S L^{o}=\sum_{p, h, t, r} \sum_{i, b}\left(\frac{\left(d d_{o}-h\right) Y_{i b}^{o p h t r}}{s l_{i b}}\right), \quad \forall o
$$

Where $d d_{o}$ is the due date expected for the order proposal $o$. Consequently, the remaining shelf life $\left(R S L^{\circ}\right)$ as:

$$
R S L^{o}=1-L S L^{o} \quad, \quad \forall o
$$

Figure 1 shows a short example about the way of calculate Eqs.(3) and (4). 


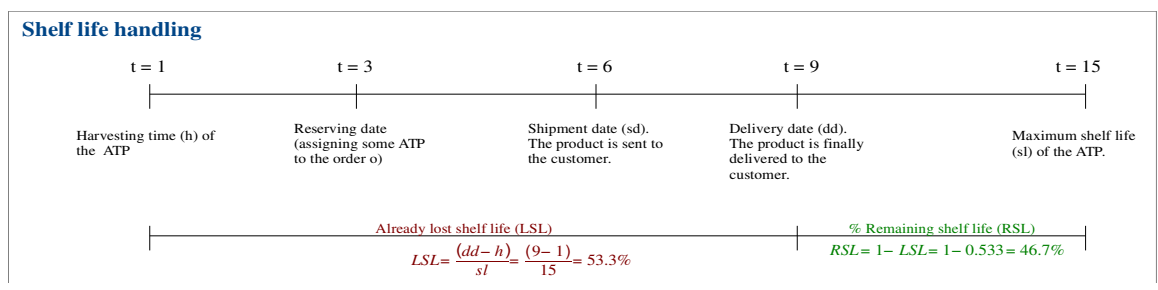

Fig.1 Example of shelf life calculation

\section{Shelf Life-based Pricing Policy}

It is usual in fruit SCs that products maintain their initial classification over time once classified and packed. This means that no quality level changes are considered. Instead, the selling price of the product is adjusted according to the aging process until the delivery date. This adjustment is made on a discrete basis, where prices are set based on predefined remaining SL intervals.

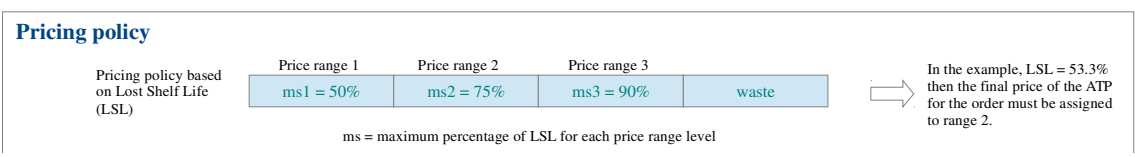

Fig.2 Pricing policy schema

Let us consider the example in Figure (2), where there are three price ranges of the ATP based on three predefined limits of the already lost shelf life (ms), and a fourth limit where the product is considered waste. Then, if the product has not overpass the first limit of the price range 1 (ms1), the selling price applied will be the highest one. With time passing the product gradually loses part of its SL until reaching the second limit. When overpassing this second limit, the selling price applied should be the second one and so on, until the product becomes waste. Therefore, the SL-based pricing policy for the assignation of homogeneous ATP should include as minimum, the following components:
$m s^{r}$
Maximum percentage of $L S L^{o}$ for each price range level $\mathrm{r}$. These percentages are defined when the price of ATP lowers from lower ranges to higher ones.
$s p_{i b}^{r} \quad$ Selling price of product $\mathrm{i}$, belonging to the subtype $\mathrm{b}$, according to price range $\mathrm{r}$.

Maximise

$$
\sum_{o, i, b} \sum_{p, h, t, r} r q_{i b}^{o} s p_{i b}^{r} Y_{i b}^{o p h t r}
$$

Subject to: Eqs. (1) - (2) and

$$
\begin{gathered}
L S L^{o}=\sum_{p, h, t, r i, b} \sum_{i, b}\left(\frac{\left(d d_{o}-h\right) Y_{i b}^{o p h t r}}{s l_{i b}}\right), \quad \forall o \\
L S L^{o}-m s^{r} \leq 1-\sum_{p, h, t, i, b} Y_{i b}^{o p h t r} \quad, \quad \forall o, r
\end{gathered}
$$




$$
\begin{gathered}
m s^{r}-L S L^{o} \leq 1-\sum_{p, h, t, i, b, r, r+1} Y_{i p h}^{\text {ophtr }} \quad, \quad \forall o, r<n r \\
m s^{r} \geq L S L^{o} \quad, \quad \forall o, r=n r
\end{gathered}
$$

In the above model, Eq. (5) aims to maximise the total sells of the product. Eqs. (1) and (2) specify the computation of the available to promise, while Eq. (6) calculates the remaining SL for the product used to fulfill each specific incoming order. Eqs. (7) and (8) oblige the binary variable to set just one price range for the subtype of product used to fulfil each specific order. Finally, Eq. (9) establishes that product used to fulfil orders cannot exceed the last price range, otherwise it becomes waste.

\section{Numerical Example}

The objective of the numerical example presented in this section is twofold. On one hand, it is shown the utility of the above equations for computing the incomes in case the product price depends on the product's freshness. On the other hand, it is shown that only maximizing incomes through serving customers with the most fresh product at the best price, presents the risk of increasing the waste. This could make impossible to serve future incoming orders. Let us assume the case of one packing plant that has to process four production lots of just one fruit (master plan) during the planning horizon composed by eight periods of time. Each lot is classified into 3 subtypes (b1, $\mathrm{b} 2, \mathrm{~b} 3$ ) according to the $\beta_{i b}^{p t}$ parameter (Table 1).
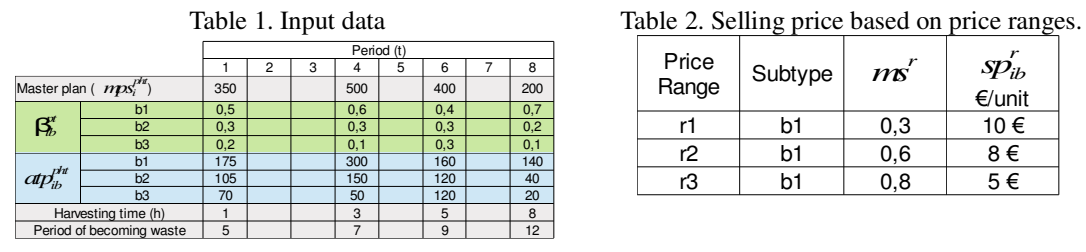

At the beginning of the OPP, we assume that no order has been already committed. Suppose a maximum shelf life of 5 periods for all subtype, a pricing policy with 3 ranges (Table 2) and cost of $5 €$ per unit of product expired. Based on the harvesting time, the SL, the last price range, and Eq. (4), it is possible to compute the period at which each lot becomes waste (Table 1). This means that the ATP quantities derived from each lot may only be used to serve orders with a due date before the period at which it expires. Furthermore, due to customer homogeneity requirements, it is not possible to accumulate discrete ATP to serve the same order (Eqs. (1)-(2)). Suppose the sequential arrival of five customer order proposals requesting certain quantities of subtype b1 for different due dates. It is our aim to analyse the results of committing these orders on-line under two different policies: a) to serve orders with the freshest product (Table 3), that is equivalent to sell the product at the highest price range and b) to serve orders with the less fresh product (Table 4). To implement the first policy, it is necessary to reserve the product from the ATP quantity as near as possible from 
the due date of the order. The column "Lot" in tables 3 and 4, show the value of the lot in which the corresponding variable $Y_{i b}^{\text {ophtr }}$ should have a value of 1 . The huge issue in fruit supply chains is that the effect of the non homogeneous product combined with the perishability, brings out an enormous risk of having waste (since the remains of the ATP cannot be mixed in order to have more quantity to fulfill new orders). As it can be seen in Table 3, the first policy maximizes the incomes per order but at the same time presents two important drawbacks. First, it is not possible to serve the last order because there is not enough unexpired homogeneous product. Second, if we assume that no more orders arrive during the planning horizon, the remaining ATP of periods 1 and 4 will expire in periods 5 and 7, respectively. Consequently, this first policy originates a negligible waste of 300 units with a total cost of $€ 1.500$.

Table 3. Serving orders from the freshest product (highest price range/Last-In-First-Out)

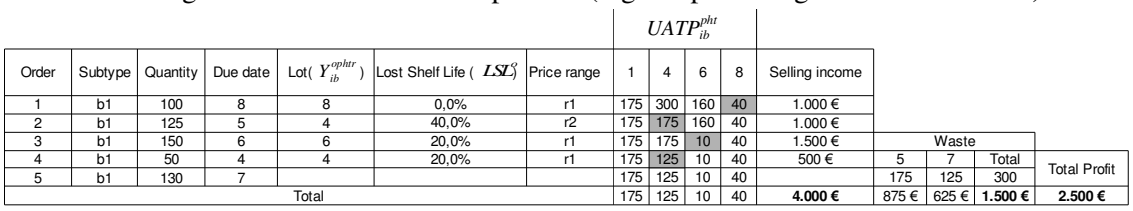

On the contrary, in order to implement the second policy it is necessary to reserve product from the ATP as far as possible from the due date, but without being expired (Table 4). Through this second policy, it is possible to serve all customer order proposals. Although the incomes per sales are lower as compared with the first policy, the total profit is higher for the second policy because the waste is considerably reduced (only 20 units for a total cost of $€ 100$ ).

Table 4. Serving order from the less fresh product (lowest price range/First-In-First-Out)

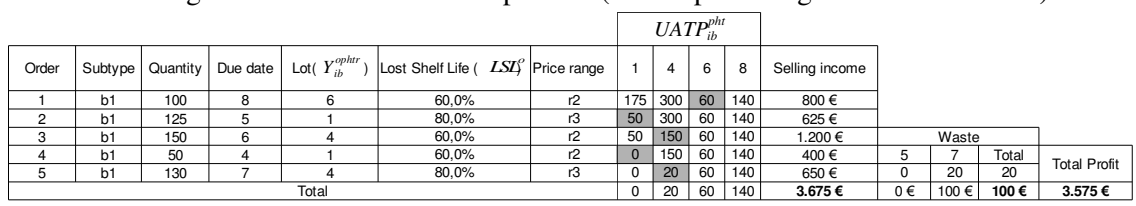

Then, there is a conflictive situation when deciding on the best balance between being careful in sell out the product as it is near to the maximum shelf life, knowing that it implies to lose a portion of profit, and achieving higher incomes by selling more fresh product but with the risk of not being able to serve some future orders and having more waste. This conflictive decision (due to the LHP, the perishability, and the expected selling prices linked with the shelf life), and the uncertainty involved in its solution, are important issues during the OPP optimization in fruit supply chains. 


\section{Conclusion}

To better analyse fruit supply chains and the corresponding order promising process, it is needed to consider the non homogeneity in the product as an inherent characteristic from nature. Subdivision of the ATP into homogeneous sublots has to be performed. Another factor is the perishability that makes even more complicated the analysis because of the risk of having wasted product. Since the customer normally need fresher product, then the pricing policy can be adjusted to the shelf life. This paper describes an approach for modelling the splitting of the ATP into homogeneous sublots can be done, how to calculate the shelf life of the product at the moment of delivery, and based on that, an expected selling price. A numerical example shows a strong conflictive situation when the OPP must decide between the prioritization between profit (as a balance of sells income and waste cost) and the customer service (as a requirement of always have fresh and homogeneous product). To better analyse this conflictive situation, uncertainty methods could be used in future research work.

\section{Acknowledgement}

This research has been supported by the Ministry of Science, Technology and Telecommunications, government of Costa Rica (MICITT), through the program of innovation and human capital for competitiveness (PINN)( PED-019-2015-1).

\section{References}

Alarcon, F., Alemany, M.M.E., Lario, F.C., Oltra, R.F., 2011. The lack of homogeneity in the product (LHP) in the ceramic tile industry and its impact on the reallocation of inventories. Boletin Soc. Espanola Ceram. Vidr. 50, 49-57. doi:10.3989/cyv.072011

Alemany, M.M.E., Grillo, H., Ortiz, A., Fuertes-Miquel, V.S., 2015. A fuzzy model for shortage planning under uncertainty due to lack of homogeneity in planned production lots. Appl. Math. Model. doi:10.1016/j.apm.2014.12.057

Alemany, M.M.E., Lario, F.-C., Ortiz, A., Gomez, F., 2013. Available-To-Promise modeling for multi-plant manufacturing characterized by lack of homogeneity in the product: An illustration of a ceramic case. Appl. Math. Model. 37, 3380-3398. doi:10.1016/j.apm.2012.07.022

Blanco, A.M., Masini, G., Petracci, N., Bandoni, J.A., 2005. Operations management of a packaging plant in the fruit industry. J. Food Eng. 70, 299-307. doi:10.1016/j.jfoodeng.2004.05.075

Grillo, H., Alemany, M.M.E., Ortiz, A., 2016. A review of mathematical models for supporting the order promising process under Lack of Homogeneity in Product and other sources of uncertainty. Comput. Ind. Eng. 91, 239-261.

Kilic, O.A., van Donk, D.P., Wijngaard, J., Tarim, S.A., 2010. Order acceptance in food processing systems with random raw material requirements. Spectr. 32, 905-925. doi:10.1007/s00291-010-0213-4 
Lin, J.T., Hong, I.H., Wu, C.H., Wang, K.S., 2010. A model for batch available-to-promise in order fulfillment processes for TFT-LCD production chains. Comput. Ind. Eng. 59, 720-729. doi:10.1016/j.cie.2010.07.026

Maihami, R., Karimi, B., 2014. Optimizing the pricing and replenishment policy for noninstantaneous deteriorating items with stochastic demand and promotional efforts. Comput. Oper. Res. 51, 302-312. doi:10.1016/j.cor.2014.05.022

Mundi, M.I., Alemany, M.M.E., Poler, R., Fuertes-Miquel, V.S., 2015. Fuzzy sets to model master production effectively in Make to Stock companies with Lack of Homogeneity in the Product. Fuzzy Sets Syst.

Tsao, Y.-C., Sheen, G.-J., 2008. Dynamic pricing, promotion and replenishment policies for a deteriorating item under permissible delay in payments. Part Spec. Issue Top. Real-Time Supply Chain Manag. 35, 3562-3580. doi:10.1016/j.cor.2007.01.024 